\title{
Percepción de los estudiantes de pregrado de Medicina sobre sus habilidades para la investigación
}

\author{
Perception of undergraduate medical students about their research skills
}

\section{Sr. Editor:}

Los estudiantes de medicina no han desarrollado las competencias necesarias para realizar un adecuado trabajo de investigación al final de sus estudios universitarios de pregrado ${ }^{(1)}$. Desde el año 2014 se reporta una frecuencia de publicación en revistas científicas de menos del $5 \%$ por alumnos de pregrado de medicina de una universidad peruana ${ }^{(2)}$. Por su parte, se ha descrito en Bolivia en el año 2018 que hasta $80 \%$ de profesionales de la salud, no habían redactado algún artículo de investigación, debido, entre otras causas, a deficiencias en conocimientos sobre metodología de investigación y escritura de un artículo ${ }^{(3)}$.

A pesar de que todas las escuelas y facultades de medicina en el Perú tienen cursos de investigación durante la carrera ${ }^{(4)}$, estos cursos no necesariamente aseguran la adquisición de estas habilidades, sobre todo si es que no existen muchos estímulos para desarrollarlas. Si bien la Ley Universitaria Peruana establece que uno de los requisitos para la obtención del título profesional es la aprobación de un trabajo de investigación o tesis ${ }^{(5)}$, algunos alumnos pueden considerar a la investigación solamente como un objetivo académico y no como fuente de adquisición de esta habilidad para promover el desarrollo científico (6).

En base a esto, exploramos en diciembre de 2019 las habilidades de investigación que algunos estudiantes de pregrado percibían que habían logrado al culminar el 5to año de medicina en la Universidad Peruana Cayetano Heredia (UPCH), Lima, Perú. Encuestamos 66 estudiantes (49,5\% mujeres, 22,5 años en promedio) que habían culminado un curso de clínicas médicas ese año ( $47 \%$ de tasa de respuesta). Los resultados se detallan en la tabla 1, donde observamos una distribución desigual de la percepción sobre las habilidades para lograr una investigación científica entre estos alumnos.

Aunque nosotros segmentamos de modo arbitrario las habilidades de investigación percibidas por los alumnos, las hemos ordenado en la tabla de acuerdo con el proceso sistemático de una investigación, de manera que pudiéramos detectar en qué parte del proceso estarían las mayores deficiencias, y así tomar mejores medidas correctivas. La mayor deficiencia percibida fue en el "análisis estadístico de una base de datos", pues casi $20 \%$ de encuestados reportaron no tener ninguna habilidad para ello. También se evidencia que la mayoría de los estudiantes considera tener un nivel bajo de habilidades en todos los pasos para realizar una investigación, con la excepción de la habilidad de búsqueda bibliográfica.

Estos resultados podrían relacionarse a que $62,6 \%$ de 32 escuelas de medicina en Perú sólo tienen uno o dos cursos de investigación a lo largo de sus programas de estudios ${ }^{(4)}$. Como menciona García ${ }^{(1)}$, si las habilidades investigativas fueran transversales y formaran parte de todos los programas a lo largo de toda la carrera de medicina, se permitiría un seguimiento y evaluación más constante del estudiante, y con ello podríamos corregir oportunamente las deficiencias percibidas en los resultados que presentamos.

No debe olvidarse también que muchas veces el objetivo final de realizar una investigación completa en medicina es lograr un producto: escribir un artículo publicable en una revista científica. Sin embargo, apenas $6 \%$ de los estudiantes que encuestamos 
Tabla 1. Número y porcentaje de estudiantes según percepción de adquisición de las habilidades de investigación.

\begin{tabular}{lcccc}
\hline Habilidades para el proceso de Investigación & \multicolumn{3}{c}{$\begin{array}{c}\text { Alumos según el grado de logro obtenido (*) al final } \\
\text { del 5to año de pregrado - n (\%) }\end{array}$} \\
\hline Realizar Búsqueda Bibliográfica & Ninguno & Bajo & Alto & Completo \\
\hline Plantear la pregunta de investigación & $0(0 \%)$ & $31(47 \%)$ & $25(37,9 \%)$ & $10(15,1 \%)$ \\
Redactar la hipótesis de investigación & $1(1,5 \%)$ & $40(60,6 \%)$ & $17(25,8 \%)$ & $8(12,1 \%)$ \\
Redactar el objetivo de investigación & $1(1,5 \%)$ & $39(59,1 \%)$ & $18(27,3 \%)$ & $8(12,1 \%)$ \\
Elegir la metodología de investigación & $0(0 \%)$ & $39(59,1 \%)$ & $19(28,8 \%)$ & $8(12,1 \%)$ \\
Seleccionar población, muestra y tipo de muestreo & $1(1,5 \%)$ & $38(57,6 \%)$ & $19(28,8 \%)$ & $8(12,1 \%)$ \\
Diseñar instrumento de recolección de datos & $2(3,0 \%)$ & $43(65,1 \%)$ & $17(25,8 \%)$ & $4(6,1 \%)$ \\
Elaborar, editar y manejar base de datos & $5(7,6 \%)$ & $46(69,6 \%)$ & $11(16,7 \%)$ & $4(6,1 \%)$ \\
Realizar análisis estadístico de base de datos & $8(12,1 \%)$ & $43(65,1 \%)$ & $11(16,7 \%)$ & $4(6,1 \%)$ \\
Interpretar resultados y redactar la discusión & $11(16,7 \%)$ & $45(68,2 \%)$ & $7(10,6 \%)$ & $3(4,5 \%)$ \\
Elaborar conclusiones y recomendaciones & $6(9,1 \%)$ & $43(65,1 \%)$ & $13(19,7 \%)$ & $4(6,1 \%)$ \\
Redactar artículo científico & $3(4,5 \%)$ & $41(62,1 \%)$ & $16(24,3 \%)$ & $6(9,1 \%)$ \\
\hline
\end{tabular}

(*) Logro obtenido de la habilidad: Ningún logro (0\%), Bajo (1-50\%), Alto (51-99\%), Completo (100\%)

consideran haber obtenido la habilidad completa para redactar un artículo científico. Por esto, consideramos que una manera objetiva de evaluar la capacidad investigativa en un estudiante de medicina podría ser mediante la publicación de una investigación propia en una revista científica; tal como proponen diversos autores ${ }^{(7)}$.

Con esto, consideramos que los estudiantes de pregrado que se expongan a esta experiencia podrán resolver otra de las dificultades reportadas en el estudio de Caron Estrada: el desconocimiento del proceso de publicación de artículos de investigación (3). Esto puede realizarse no solo mediante la redacción de investigaciones originales, sino también de cartas al editor o reportes de casos, las cuales deben enviarse a revistas científicas indexadas e, idealmente, ser publicadas antes de terminar su carrera en pregrado. Al presentar sus trabajos a una revista científica, tendrán que leer y cumplir las normas de publicación para autores que tienen cada una de ellas, lo que les hará ver que el proceso de publicación no es tan difícil como se cree. Todo esto podría ayudar a que el logro de la habilidad completa para desarrollar una investigación sea la regla y no la excepción al final de la carrera.

$$
\begin{array}{r}
\text { Otto Barnaby Guillén-López }{ }^{1,2, a}, \\
\text { Jimy Henry Álvarez-Mayorga } \\
\text { Diana Elizabeth Calle-Jacinto de Guillén }
\end{array}
$$

\section{Correspondencia:}

Otto Barnaby Guillén-López

Dirección: Avenida Antonio Miroquesada 572, Dpto. 1802. Magdalena del Mar, Lima-Perú.

Correo electrónico: otto.guillen.1@upch.pe

Teléfono. $+51-997851413$

\footnotetext{
Universidad Peruana Cayetano Heredia. Lima, Perú.

Hospital Nacional Arzobispo Loayza. Lima, Perú.

Hospital Santa Rosa. Lima, Perú.

Médico especialista en Medicina Interna;

Médico Cirujano;

Magíster en Gestión del Cuidado en Enfermería;
} 


\section{REFERENCIAS BIBLIOGRÁFICAS}

1. García M. La investigación en los planes de estudio: una necesidad olvidada. Educ Med. 2020; 21(5):283284. DOI: 10.1016/j.edumed.2020.09.0031.

2. Mejia MO, Veramendi-Espinoza L, Huerta-Collado YM, Montenegro-Idrogo JJ. Baja publicación de investigaciones médico estudiantiles curriculares de una universidad peruana. Rev Peru Med Exp Salud Publica. 2014; 31(3):608.

3. Caron R, Mattos P, Barboza JJ. Dificultades para la elaboración de artículos de investigación científica en estudiantes de posgrado en salud. Educ. med. super. 2020; 34(3):e1624. (Citado el 8 de enero del 2021) Disponible en: http://www.ems.sld.cu/index. $\mathrm{php} / \mathrm{ems} /$ article/view/1624

4. Taype-Rondán, Á, Huaccho-Rojas J, Pereyra-Elías R, Mejía CR, Mayta-Tristán, P. Características de los cursos de investigación en escuelas de medicina del
Perú. Arch Med. 2015; 11 (2):1-7. DOI: 10.3823/1243

Ministerio de Educación - Perú. Ley Universitaria ${ }^{\mathrm{a}}$ 30220. (Citado el 6 de agosto del 2021) Disponible en: http://www.minedu.gob.pe/reforma-universitaria/ pdf/ley_universitaria.pdf

5. Osada J, Ruiz-Grosso P, Ramos M. Estudiantes de pregrado: el futuro de la investigación. Rev Peru Med Exp Salud Publica. 2010; 27(2). DOI: 10.17843/ rpmesp.2010.272.1485

6. Alarco JJ, Changllio-Calle G, Cahuana-Salazar M. Investigación en pregrado: interés según sexo y ciclo académico. Educ. Med 2017; 18(1):67-73. DOI: 10.1016/j.edumed.2016.04.004

Recibido: $15 / 03 / 2021$

Aceptado: 26/08/2021 